\title{
Organización del inventario en las empresas filiales de PDVSA industrial
}

Organization of the inventory in the subsidiary companies of PDVSA industrial

Nelson Cabrita

cabritanj@gmail.com

Código ORCID: 0000-0002-8470-7745

Universidad del Zulia - Venezuela
RESUMEN

Se analizó el proceso de organización de inventario en las empresas filiales de PDVSA Industrial, bajo una investigación descriptiva, con diseño de campo, no experimental y transeccional. La población estuvo conformada por los gerentes, líderes y supervisores de las empresas filiales de PDVSA Industrial, ubicadas en los municipios Cabimas y Lagunillas de la Costa Oriental del Lago. Para la recolección de datos se empleó la encuesta mediante un cuestionario, conformado por 9 ítems con escala de frecuencia. La validez se realizó a través del juicio de expertos, y para calcular su confiabilidad se empleó el método del Coeficiente Alfa de Cronbach, cuyo resultado fue de 0,907. El análisis de los datos se realizó mediante la estadística descriptiva, con base en la media aritmética. Se verifico en conjunto una moderada aplicación de la selección de personal, capacitación y proveedores como actividades involucradas en el proceso de organización de inventario.

Palabras clave: Capacitación; inventario; proceso de organización; proveedores; selección de personal

The inventory organization process in the PDVSA Industrial affiliate companies was analyzed, under a descriptive investigation, with a field design, not experimental and transectional. The population was made up of the managers, leaders and supervisors of the PDVSA Industrial subsidiary companies, located in the Cabimas and Lagunillas municipalities of the Eastern Lake Coast. For data collection, the survey was used using a questionnaire, made up of 9 items with a frequency scale. Validity was carried out through expert judgment, and the Cronbach Alpha Coefficient method was used to calculate its reliability, with a result of 0.907 . Data analysis was performed using descriptive statistics, based on the arithmetic mean. Overall, a moderate application of the selection of personnel, training and suppliers was verified as activities involved in the inventory organization process.

Key words: Training; inventory; organization process; suppliers; personnel selection 


\section{INTRODUCCIÓN}

Actualmente y atendiendo a los altos niveles de competencia existente en los mercados mundiales, las organizaciones empresariales han venido diseñando, adoptando e implementando nuevas estrategias gerenciales de vanguardia, de forma tal que puedan desarrollar ventajas competitivas en todos los ámbitos y les permitan hacer frente a la competencia, donde cada día se hace más difícil la sobrevivencia de las organizaciones, especialmente si estas no se adaptan a las condiciones cambiantes sobrevenidas de la globalización de los mercados, los avances tecnológicos, en fin una economía mundial en constante proceso de cambio.

Es por ello que, las organizaciones para lograr mantenerse, además de avanzar están dedicando grandes esfuerzos para comprender estos cambios, así como la importancia e influencia que estos tienen en el desarrollo y funcionamiento de las organizaciones, direccionando sus esfuerzos estratégicos como gerenciales hacia el manejo efectivo de todos los procesos y actividades que permitan maximizar la eficiencia con respecto a los recursos económicos, técnicos, materiales y humanos, todo con el objetivo de minimizar el riesgo de perdidas, optimizando el manejo de los recursos.

Ante esta situación, la gestión o manejo de los inventarios tiene un papel preponderante en las organizaciones, motivado a que es una parte fundamental de la eficiencia y productividad de las mismas, principalmente en las actuales condiciones de alta competitividad de los mercados, donde es primordial el equilibrio entre los niveles bajos de inventario y los elevados niveles de servicios que garanticen la satisfacción de los usuarios, de igual forma la gestión de inventarios es considerada como una de las funciones administrativas más importantes de toda organización, ya que permite la orientación de la toma de decisiones, cambios e innovaciones en los procesos, llevando al establecimiento de un sentido de dirección y de mejora continua de la gestión de los procesos y calidad de servicios.

Sobre ello, Horngren (2004), señala que la gestión de inventario consiste en lograr la máxima productividad, mediante el balance en la inversión de activos con lo que de manera real se requiere para la fluidez de las operaciones. En este sentido, los inventarios constituyen para las organizaciones, una especie de sistema de amortiguación, que absorben tanto los errores de planeación, así como las variaciones o fluctuaciones no previstas en la oferta y la demanda, de forma tal que facilita el flujo de las operaciones de producción y mercadeo.

De igual forma, la gestión de inventarios, según lo indica Ferrín (2007), busca controlar las existencias de materias primas y productos terminados en el almacén, de manera que no se produzcan roturas en el stock que dificulten los procesos productivos o las ventas; ni excesos en los stocks causantes de material obsoleto, costos adicionales y cifras de inventarios mayores a las necesarias, empleando las técnicas y herramientas propias de la gestión y de previsión de la demanda.

Lo mencionado anteriormente implica, que deben considerarse la aplicación de herramientas de planificación y de controles preventivos y detección de sistemas de inventario, con la finalidad de minimizar los costos totales en las actividades de abastecimiento, garantizando los niveles de atención eficiente a los usuarios para dar cumplimiento con la planificación y desarrollo de materiales bajo una filosofía de poseer almacén disponible en el mercado. 
Con base en las ideas expuestas, se hace necesario la aplicación y mejora continua de la gestión de inventario, que produzcan y promuevan el impulso de la organización, contribuyendo así a mejorar su posición en el mercado, permitiendo minimizar los niveles de incertidumbre, y que con la aplicación de procesos adecuados permitan y faciliten el manejo y control de las existencias administrativas y físicas, logrando dar así consistencia al desempeño de la organización. La gestión de inventarios tiene un decisivo impacto en las estructuras de costos de cualquier organización, por lo que tener un estricto control sobre estos costos, proporcionara un importante instrumento de decisión a la dirección, el cual permitirá mejorar los niveles de gestión de los procesos y la calidad de los servicios.

Por tal motivo, a criterio del investigador una buena administración de los inventarios es esencial para el funcionamiento exitoso de las organizaciones, resultando ser una de las áreas donde es más factible la reducción de costos sin reducir los ingresos, elemento este de vital importancia para la subsistencia de la organización en los tiempos modernos. De allí, la importancia de estructurar y aplicar en todos los negocios este concepto para ser competitivos en el desarrollo de sus actividades.

Al respecto PDVSA Industrial, S.A., filial no petrolera de la Corporación Petróleos de Venezuela, S.A., creada en 2007 con la finalidad de desarrollar el sector industrial del país, y que actualmente abarca 118 unidades de producción, organizadas en 28 empresas filiales y seis grupos industriales en todo el territorio nacional, no escapa a esta realidad, llegando incluso a ser de las más afectadas ya que por sus grandes estructuras organizacionales, el manejar grandes niveles de inventarios incrementan de manera considerable sus costos, lo que representa millones de dólares, pero el manejar niveles insuficientes de inventarios o stock, impacta de forma negativa sus operaciones, ya que podrían no satisfacer sus necesidades de operación; por estas razones PDVSA Industrial, S.A., y sus empresas filiales necesitan disponer de niveles óptimos de inventarios para satisfacer sus demandas, garantizando la continuidad de sus operaciones y procesos de producción con altos niveles de eficiencia.

En la actualidad, PDVSA Industrial, S.A., y sus filiales cuentan con gerencias especializadas en la procura de bienes para el sector industrial, bajo una estrategia corporativa alineada con los planes y políticas de la nación, que incluye el trabajo en conjunto con las unidades usuarias para satisfacer sus necesidades. Estas gerencias son conocidas a nivel nacional como las gerencias de procura de PDVSA Industrial, S.A., y sus filiales, y están ubicadas en diversas aéreas del territorio nacional.

Mediante información obtenida de indicadores de gestión empleados por las gerencias responsables, entrevistas no estructuradas así como la observación directa por parte del investigador, se pudo constatar que en la actualidad los procesos de manejo de los inventarios y almacenes de las empresas filiales de PDVSA Industrial, S.A., en todo el país evidencian debilidades, fallas e inexistencia en algunos casos de mecanismos de planificación y control de las existencias que permitan detectar oportunamente las desviaciones, que afectan el normal desarrollo de las operaciones.

De acuerdo a lo planteado anteriormente, la situación en las empresas filiales de PDVSA Industrial, S.A., en la región zuliana, no escapan a esta realidad, tal son los casos de las ubicadas en los municipios de la Costa Oriental del Lago de Maracaibo, específicamente las empresas Venezolana Socialista del Plástico (Vensoplast), 
Diques y Astilleros Industriales (DDAA), Empresa Nacional de Tubulares (ENATUB).

De continuar esta situación, podrían presentarse fallas más graves en los sistemas de gestión de inventarios de las empresas filiales de PDVSA Industrial, S.A., lo que repercutiría con gran impacto en las estructuras funcionales y de operación de las mismas, afectando el normal desarrollo de las operaciones si no se dispone del material, equipos, herramientas, y de un personal capacitado que permitan elevar, con la organización un adecuado control y manejo de los inventarios.

\section{Proceso de organización de inventario}

Dada la importancia del inventario y su estrecha relación con los procesos productivos Chase y otros (2009), lo definen como la cantidad de existencias de un bien o recurso cualquiera, usado por las empresas. De igual manera, el uso de inventarios permite manejar eficientemente los materiales a través del proceso de organización.

En este tal sentido, para Thomas (2004), la organización es la función administrativa de ensamblar y coordinar los recursos humanos, financieros, físicos, de información y otros que sean necesarios para lograr las metas. A su vez indica, que son actividades que incluyan atraer gente a la organización, especificar las responsabilidades del puesto, agrupar tareas en unidades de trabajo, dirigir y distribuir recursos y crear condiciones para que las personas y las cosas funcionen en conjunto para alcanzar el máximo éxito.

Desde la perspectiva del inventario, la organización se refiere a todos los procesos que coadyuvan al suministro, accesibilidad y almacenamiento de productos en alguna compañía para minimizar los tiempos y costos relacionados con el manejo del mismo: es un mecanismo a través del cual, se administra de manera eficiente el movimiento $y$ almacenamiento de mercancía, así como el flujo de información y recursos que resultan de ello. Involucra distintos aspectos, pero en términos generales se subdivide en lo correspondiente a selección de personal, capacitación y proveedores.

Visto así, se analiza acerca de la organización para las empresas filiales de PDVSA Industrial S.A., en cuanto a su importancia para la gestión de inventarios, las implicaciones que tiene el proceso de diseñar los cargos y asignación de labores en manejo de materiales. En tal sentido, una selección adecuada junto a una capacitación en inventario permite el logro de los objetivos de la empresa, además permite que los recursos humanos su ubicación en el puesto adecuado, de acuerdo a sus habilidades desarrolladas. Así mismo, desarrolla en los trabajadores las herramientas necesarias para una evaluación de los proveedores para el suministro de materiales.

\section{Selección de personal}

Es importante destacar que el administrador financiero no tiene el control principal sobre la administración de los inventarios, sin embargo, su función es necesaria ya que es quien maneja los recursos financieros para su adquisición. Por ello seleccionar un personal adecuado que maneje con eficiencia el stock de las mercancías es primordial y esto es debido a que existe controversia entre las diferentes áreas funcionales de la empresa referente a los niveles adecuados de inventarios que debe manejarse.

En este sentido, para el gerente financiero se debe manejar niveles bajos de inventario, pues se trata de un activo corriente que presenta menos rentabilidad que los activos no corrientes. Para el gerente de marketing se debe manejar grandes cantidades de inventario de productos 
terminados para atender eficazmente a los consumidores. El gerente de producción requiere tener elevados niveles de inventario de materia prima para la producción y de productos terminados para hacer las entregas a tiempo que solicite el gerente de comercialización. Mientras, el gerente de compra prefiere adquirir grandes cantidades de materia prima para obtener mejores descuentos por las adquisiciones al por mayor. En tal sentido, seleccionar un personal idóneo que logre equilibrar el inventario de cada área representa un proceso de organización relevante para cualquier empresa.

Desde esta perspectiva, Chiavenato (2009), conceptualiza seleccionar al personal como el proceso de escoger al mejor candidato para un área de la empresa, cumpliendo de ese modo, la función de filtro que sólo permite la entrada a algunas personas con características beneficiosas para la organización. Seleccionar al personal tiene como finalidad buscar entre diversos candidatos, el más adecuado para desempeñar funciones inherentes a un determinado departamento.

El criterio para la selección es el resultado de la comparación de dos variables; por un lado, los requisitos del puesto de trabajo y por el otro, el perfil de las características de los candidatos que se presentan para disputarlos. Una vez, obtenida la información respecto al puesto a ocupar $\mathrm{O}$ las competencias requeridas, el siguiente paso es conocer y verificar la información que describe el perfil de los postulantes al empleo.

Sobre la base de las ideas expuestas, las empresas filiales de PDVSA Industrial, S.A., les resulta conveniente considerar en el proceso de selección de personal con conocimientos de inventario, ello implica una serie de pasos que van desde escoger el candidato con mayor probabilidades de cumplir con el perfil de cargo e idóneos para cubrir una vacante tomando como parámetro las necesidades del departamento. Las decisiones a tomar en cuanto a la selección deben hacerse con un análisis tomando en cuenta las características y el grado de habilidades del aspirante al cargo.

\section{Capacitación}

Las empresas invierten gran parte de sus recursos económicos en su inventario, por lo que resulta clave para el que se designe a una persona capacitada para gestionarlo, alguien con el conocimiento y experiencia suficiente para tomar decisiones estratégicas que sirvan para optimizar su control.

En este sentido, para Newstrom (2007), la capacitación es el procedimiento orientado a mejorar el rendimiento en el puesto de trabajo, permitiendo de esa manera a los trabajadores habilidades para la toma de decisión acertadas de acuerdo al contexto donde se manejen. La capacitación es la clave para el incremento de la productividad y mejore el servicio al cliente, es importante desarrollar habilidades en calidad, inventarios justo a tiempo, reingeniería de procesos y control estadístico.

Por otra parte, el coaching es una manera adecuada de ayudar a los trabajadores a aprender, un supervisor del coach se mantiene atento a las oportunidades de mejoramiento, formula preguntas para estimular el pensamiento del empleado. En ese sentido, se le da al empleado herramientas para la toma de decisiones a través de la observación, demostración, interrogación y retroalimentación oportuna de la supervisión.

Para Chiavenato (2009, p.371), la capacitación "es un medio para apalancar el desempeño en el trabajo, siendo el proceso mediante el cual se prepara a la persona para que desempeñe con excelencia las tareas 
específicas del puesto que ocupa". Desarrollando competencias en los sujetos para que sean más productivas, creativas e innovadoras con el propósito de cumplir con las políticas y los objetivos de la organización.

La capacitación les genera valor a las personas orientándose a las necesidades actuales del negocio, pretendiendo mejorar las habilidades con el desempeño inmediato del trabajo. Modificando actitudes y comportamientos diferentes para ser más eficaces en sus puestos de trabajo, de igual manera, los programas de capacitación buscan el desarrollo de nuevos hábitos para cumplir con los clientes internos o externos, así como, con los subordinados y con la empresa.

Visto así, la capacitación para las empresas filiales de PDVSA Industrial, S.A., es de suma importancia para la generación de conocimientos de los trabajadores en el desarrollo de habilidades, capacidades con la finalidad de cambiar actitudes, contribuyendo de ese modo, con el incremento de la productividad y al cumplimiento de los objetivos de la empresa. La capacitación es una herramienta fundamental porque gestiona de una manera organizada la modificación de las conductas del personal de acuerdo a las necesidades de la organización.

\section{Proveedores}

La importancia de contar con un buen proveedor radica en la posibilidad de alcanzar márgenes de utilidad más altos y brindar un excelente servicio a los clientes, con ello se evita retrasos en las entregas $u$ obtener mercancía en malas condiciones. Si los artículos no están disponibles a tiempo, porque el proveedor no los entregó cuando debía, es muy probable que los compradores busquen otra opción
En este sentido, Velásquez (2004), sostiene que una de las funciones del sistema de compras es la de proveer de lo necesario para las operaciones de la organización y obviamente debe tener una relación directa con sus proveedores. Es así que sugiere la necesidad de crear un clima de buena voluntad para la organización, mediante unas relaciones comerciales cordiales, manteniendo y desarrollando una selección de proveedores acorde a sus necesidades de producción para obtener el mejor valor en los insumos adquiridos y al precio más bajo posible.

De igual manera, Weitz y otros (2005), señalan la importancia de evaluar los proveedores potenciales, de acuerdo a sus propuestas presentadas y el funcionamiento de los productos comprados. El grado de conocimiento de los trabajadores de los proveedores con los cuales cuenta la empresa va a incidir en la toma de decisiones para la selección de los más adecuados, es por ello, que afectan la evaluación, selección de los productos; dado a las necesidades racionales, relacionadas en forma directa con el funcionamiento del producto con las necesidades emocionales.

Visto así, a efectos de la investigación, los proveedores se ocupan de gestionar la relación con los suministradores de servicios de los que depende la organización. Su principal objetivo es alcanzar la mayor calidad a un precio adecuado, para ello debe obtener la información relacionada con los proveedores y los servicios que estos prestan. En tal sentido, las empresas filiales de PDVSA Industrial, S.A., deben disponer de un registro de proveedores, que garantice el suministro de materiales $\mathrm{o}$ insumos, de acuerdo a sus necesidades y en las condiciones previamente definidas en el proceso de negociación. Ante 
esta situación, la organización debe considerar en sus políticas y estrategias, una base de proveedores amplia que garantice su existencia en los almacenes para un determinado periodo de tiempo.

\section{MATERIALES Y MÉTODO}

La metodología utilizada fue de tipo descriptiva, con diseño de campo, no experimental y transeccional. La población estuvo conformada por las empresas filiales de PDVSA Industrial, en la Costa Oriental del Lago, específicamente las ubicadas en los municipios Cabimas y Lagunillas, identificadas como: Venezolana Socialista del Plástico (Vensoplast), Diques y Astilleros Industriales (DDAA), Empresa Nacional de Tubulares (ENATUB); cuyos sujetos informantes fueron los gerentes, líderes y supervisores, así la población objeto de estudio quedó conformada por 18 personas: 3 gerentes, 4 líderes y 11 supervisores de las empresas mencionadas.

Para la recolección de datos se empleó la encuesta mediante la aplicación de un cuestionario, el mismo quedó conformado por 9 ítems con escala tipo de frecuencia. La validez se realizó a través del juicio de 5 expertos, y para calcular su confiabilidad se empleó el método del Coeficiente Alfa de Cronbach, cuyo resultado fue de 0,907, lo cual indicó muy alta confiabilidad.

El análisis de los datos se realizó mediante la estadística descriptiva, con base en la media aritmética. Para tal efecto, el investigador diseñó un baremo para el análisis de la media aritmética, en el cual se muestra la alternativa de respuesta, intervalo y categoría asignada, tal como se muestra en el tabla 1.

Tabla 1. Baremo para la interpretación de la media aritmética

\begin{tabular}{cccc}
\hline Alternativa & Puntaje & Intervalo & Categoría \\
\hline Siempre & 5 & $4.21-5.00$ & Muy alta aplicación \\
Casi siempre & 4 & $3.41-4.20$ & Alta aplicación \\
Algunas veces & 3 & $2.61-3.40$ & Moderada aplicación \\
Casi nunca & 2 & $1.81-2.60$ & Baja aplicación \\
Nunca & 1 & $1.00-1.80$ & Muy baja aplicación \\
\hline
\end{tabular}

Fuente: Cabrita (2019)

\section{RESULTADOS Y DISCUSION}

Iniciando con el proceso de análisis de los resultados, la tabla 2 muestra la información recopilada sobre el indicador selección de personal, el cual presenta una media aritmética de 1,56; indicando muy baja aplicación de la selección del personal como un proceso de previsión que procura prever cuáles solicitantes tendrán éxito si se les contrata. 
Nelson Cabrita

Tabla 2. Indicador: Selección de personal

\section{En la gestión de inventario que usted coordina}

1. Se evalúa el perfil de los aspirantes considerando el conocimiento que se tenga en el manejo de inventario

2. Se selecciona al candidato que cumpla con el perfil del cargo en el área de inventario

3. Se verifican los datos que confirme el sujeto más adecuado con las características del puesto de trabajo.

\begin{tabular}{ccc} 
Ítems & Promedio & Categoría \\
1 & 1,44 & Muy baja aplicación \\
2 & 1,72 & Muy baja aplicación \\
3 & 1,50 & Muy baja aplicación \\
Promedio & $\mathbf{1 , 5 6}$ & Muy baja aplicación \\
\hline
\end{tabular}

Fuente: Cabrita (2019)

Así, en el ítem 1 se obtuvo un valor de media de 1,44 lo que refiere a una muy baja aplicación según opinión de los encuestados de que se evalúa el perfil de los aspirantes considerando el conocimiento que se tenga en el manejo de inventario. Igualmente, en el ítem 2 el valor de media de 1,72 siguiere muy baja aplicación de que se selecciona al candidato que cumpla con el perfil del cargo en el área de inventario. Por su parte, con muy baja aplicación se verifican los datos que confirme el sujeto más adecuado con las características del puesto de trabajo, evidenciado esto en el 1,50 de media aritmética obtenido en el ítem 3.

Basado en los resultados descritos anteriormente, a juicio del investigador, las empresas analizadas no buscan entre diversos candidatos, el más adecuado para desempeñar funciones inherente a la gestión de inventario. De manera que, al contrastar la realidad manifiesta con la teoría consultada, no existe congruencia con lo expuesto por Koontz y Weihrich (2007), para quien la selección del personal es un proceso que requiere de un análisis de las necesidades de la empresa y del puesto de trabajo.

En el mismo orden de ideas, en la tabla 3 se exponen los resultados para el indicador capacitación, el cual obtuvo en líneas generales un valor de 3,50 en su media aritmética, siendo clasificado como alta aplicación de un conjunto de conocimientos y el mejoramiento de aptitudes, capacidades, entendimiento y condiciones naturales de una persona que permite la mejora continua de las empresas analizadas. 
Tabla 3. Indicador: Capacitación

\section{En la gestión de inventario que usted coordina}

4. Se capacita en el manejo de inventarios al personal adscrito a ese departamento

5. El personal desarrolla competencias en el manejo de inventario

6. El personal cumple con las labores inherentes a su cargo desempeñado

\begin{tabular}{ccc} 
Ítems & Promedio & Categoría \\
4 & 1,61 & Muy baja aplicación \\
5 & 4,28 & Muy alta aplicación \\
6 & 4,61 & Muy alta aplicación \\
Promedio & $\mathbf{3 , 5 0}$ & Alta aplicación \\
\hline
\end{tabular}

Fuente: Cabrita (2019)

Describiendo la información arrojada por los encuestados, la media de 1,61 en el ítem 4 indica muy baja aplicación de que se capacita en el manejo de inventarios al personal adscrito, no obstante con muy alta aplicación de 4,28 como promedio en el ítem 5 asegura que el personal desarrolla competencias en el manejo de inventario; entretanto que el ítem 6 expone con muy alta aplicación que el personal cumple con las labores inherentes a su cargo desempeñado, según el valor de su media de 4,61.

En atención a esto, puede decirse bajo la perspectiva del investigador, que la capacitación es una herramienta fundamental porque gestiona de una manera organizada la modificación de las conductas del personal de acuerdo a las necesidades de la organización, en tal sentido, en las empresas analizadas, aun cuando el indicador capacitación se ubica en alta aplicación, estas deben encontrar la manera de preparar a sus trabajadores para que desempeñe con excelencia las tareas específicas del puesto que ocupa, tal como lo refiere Chiavenato (2009).

En lo que respecta al indicador proveedores se tiene una media de 3,43 , tal como lo evidencia la tabla 4; categorizándose como alta aplicación de personas o sociedades que suministran la materia prima utilizada para producir los bienes o servicios necesarios para una actividad. 
Nelson Cabrita

Tabla 4. Indicador: Proveedores
En la gestión de inventario que usted coordina
7. Los proveedores proporcionan los insumos en el tiempo establecido
8. Se dispone de un amplio número de proveedores
9. Se tiene un historial de proveedores para registrar su desempeño

\section{Ítems}

7

8

9

Promedio
Promedio

1,50

4,28

4,50

3,43

\section{Categoría}

Muy baja aplicación

Muy alta aplicación

Muy alta aplicación

Fuente: Cabrita (2019)

Como se observa, en el ítem 7 se manifiesta muy baja aplicación según opinión de los encuestados de que los proveedores proporcionan los insumos en el tiempo establecido, notándose en el valor promedio de 1,50. No obstante, en el ítem 8 se denota muy alta aplicación de que se dispone de un amplio número de proveedores, con una media obtenida de 4,28; y en el ítem 9 con muy alta aplicación se tiene un historial de proveedores para registrar su desempeño, obteniendo una media de 4,50.

Expuesto lo anterior, a juicio del investigador en las empresas filiales de PDVSA Industrial, en la Costa Oriental del Lago, a pesar de posicionarse en alta aplicación en el indicador, estas deben disponer de un registro de proveedores, que garantice el suministro de materiales $o$ insumos, de acuerdo a sus necesidades y en las condiciones previamente definidas en el proceso de negociación. Lo anteriormente planteado se sustenta en la teoría de Velásquez (2004), quien sostiene que una de las funciones del sistema de compras es la de proveer de lo necesario para las operaciones de la organización y obviamente debe tener una relación directa con sus proveedores.

Visto así, las empresas filiales de PDVSA Industrial, S.A, en la Costa Oriental del Lago, deben identificar y seleccionar los proveedores fundamentales para asegurar la continuidad del negocio y la satisfacción de los clientes, por lo tanto, el proceso de selección del proveedor es una tarea necesaria en estas empresas y requiere mantener de forma permanente un método de identificación, contacto, evaluación y gestión de la relación con el suministrador.

Finalmente, la tabla 5 resume los resultados relacionados con la dimensión proceso de organización de inventario, con un promedio de 2,83 lo que la ubica en una categoría de moderada aplicación indicando que los aspectos que se analizan en este proceso de manera moderada se cumplen según lo esperado, para alcanzar los logros en la gestión de inventario en las empresas filiales de PDVSA Industrial, en la Costa Oriental del Lago. 
Según los resultados obtenidos, se observa que las empresas en estudio con muy baja aplicación realiza un proceso idóneo para la elección del personal $(1,56)$; no obstante con alta aplicación se desarrollan las habilidades y conocimientos a fin de que los trabajadores sean capaz de sobrellevar con más afectación la ejecución de su propio trabajo $(3,50)$ y con alta aplicación se dispone de un conjunto de proveedores $(3,43)$

Tabla 5. Dimensión: Proceso de organización de inventario

\begin{tabular}{lcc}
\hline \multicolumn{1}{c}{ Indicador } & Promedio & Categoría \\
\hline Selección de personal & 1,56 & Muy baja aplicación \\
Capacitación & 3,50 & Alta aplicación \\
Proveedores & 3,43 & Alta aplicación \\
Promedio & $\mathbf{2 , 8 3}$ & Moderada aplicación \\
\hline
\end{tabular}

Fuente: Cabrita (2019)

En consecuencia, a criterio del investigador, a pesar de posicionarse la dimensión en moderada aplicación, las actividades involucradas con el proceso de organización, representan oportunidades de mejoras, donde se logre crear un mecanismo que permita a las empresas filiales de PDVSA Industrial una selección adecuada junto a una capacitación en inventario a fin de lograr sus objetivos, además de permitir que los recursos humanos ocupen el puesto adecuado, de acuerdo a sus habilidades desarrolladas. De lograrse esto, se daría validez a lo expuesto por Thomas (2004), para quien la organización es la función administrativa a través de la cual se ensamblan y coordinan los recursos humanos, financieros, físicos, de información y otros que son necesarios para lograr las metas.

\section{CONCLUSIONES}

Se analizó el proceso de organización de inventario en las empresas filiales de PDVSA
Industrial, en la Costa Oriental del Lago, destacándose que las empresas en estudio con muy baja aplicación realiza un proceso idóneo para la elección del personal; no obstante con alta aplicación se desarrollan las habilidades y conocimientos a fin de que los trabajadores sean capaz de sobrellevar con más afectación la ejecución de su propio trabajo y con alta aplicación se dispone de un conjunto de proveedores.

De manera que se verifico una moderada aplicación de las actividades involucradas en el proceso de organización, que coadyuvan al suministro, accesibilidad y almacenamiento de mercancía, así como el flujo de información y recursos que resultan de ello. Involucra distintos aspectos, pero en términos generales se subdivide en lo correspondiente a selección de personal, capacitación y proveedores. 


\section{REFERENCIAS}

Certo, S. (2001). Administración Moderna. (OCTAVA EDICION). Editorial PenticeHall. Colombia

Chase, R; Jacobs, R. y Aquilano, N. (2009). Administración de Producción y Operaciones: Manufactura y Servicios. Editorial Mc Graw Hill. Colombia

Chiavenato, I. (2009). Gestión del Talento Humano. Editorial: Mc Graw Hill. México

David, F. (2013). Conceptos de administración estratégica. Décimo cuarta edición. Pearson Educación México

Ferrin, A (2007). Gestión de stocks en la logística de materiales. Segunda Edición. Fundación Confemetal. Madrid. España

Horngren W. (2004) Matriz de Control Interno. Editorial Cangallo Barcelona España.

Koontz, H.; y Weihrich, H. (2007). Elementos de Administración. Un Enfoque Internacional. Editorial: Mc Graw Hill. México
Newstrom, J. (2007). Dirección. Gestión para lograr Resultados. Editorial: Mc Graw Hill. México

Stoner, J.., Freeman, E. y Gilbert, D. (2006). Administración. Editorial Prentice Hall Hispanoamericana, S.A. México

Thomas, M (2004). Master de Recursos Humanos. Ediciones Deusto. España

Velásquez, G. (2004). Administración de los Sistemas de Producción. Sexta Edición. Editorial: LIMUSA, Noriega Editores. Colombia

Weitz, B.; Castlewerry, S. y Tanner, J. (2005).Ventas. Construyendo Sociedades. Editorial: Mc Graw Hill. México

Wheelen, T. y Hunger, J.D. (2007).Administración estratégica y política de negocios.: Pearson Education. México 